\title{
Pretreatment nutritional status and response to checkpoint inhibitors in lung cancer
}

\author{
Chung-Shien Lee*,1,2 (D), Craig E Devoe², Xinhua Zhu², Joanna Stein Fishbein ${ }^{3}$ \& Nagashree \\ Seetharamu² \\ ${ }^{1}$ St. John's University, College of Pharmacy \& Health Sciences, Department of Clinical Health Professions, Queens, NY 11439, USA \\ ${ }^{2}$ Division of Medical Oncology \& Hematology, Northwell Health Cancer Institute, Donald \& Barbara Zucker School of Medicine at \\ Hofstra/Northwell, Lake Success, NY 11042, USA \\ ${ }^{3}$ Biostatistics Unit, The Feinstein Institutes for Medical Research, Manhasset, NY 11021, USA \\ *Author for correspondence: leec3@stjohns.edu
}

\section{Practice points}

- Out of the 106 patients analyzed, $17 \%$ of patients had a low albumin $(<3.5 \mathrm{~g} / \mathrm{dl})$ at baseline and $37.7 \%$ reported weight loss of $5 \%$ or more in the 6 months prior to starting immunotherapy.

- Low serum albumin level status, malnutrition status based on composite nutritional status, report of at least $5 \%$ weight loss within 6 months of presentation and baseline Eastern Cooperative Group performance status were each significantly associated with overall survival (OS) on univariate screen $(p<0.0001, p=0.0001, p=0.0003$ and $\mathrm{p}=0.0162$, respectively).

- Age was also marginally associated with OS on univariate analysis $(p=0.06)$.

- On multivariate analysis, an interesting U-shaped relationship was noted between age and OS.

- Risk of death was highest at lower ages, insignificant at 'middle' ages (i.e., ages 60-75 years), and at the lowest for elderly patients (i.e., $>75$ years of age).

- Patients above the estimated age of 75 years have an incrementally improved survival with every 5 year increase in age at the time of initiation of checkpoint inhibitor, whereas the risk of death increases with every 5 year increase in age from 45 to 60 years.

- Serum albumin less than 3.5 at baseline was associated with progression-free survival on univariate screen at $p<0.1$ level $(p=0.079)$

- Overall, 96 patients (90\%) experienced an adverse event, with only three patients $(2.8 \%)$ experiencing a severe event.

Background: Checkpoint inhibitors are integral to non-small-cell lung cancer treatment. Existing data suggests that nutritional status may play a role in antitumor immunity. Materials \& methods: This retrospective study of 106 non-small-cell lung cancer patients who started checkpoint inhibitors between 2014 and 2017 at our institution assessed relationship of nutritional parameters to overall survival (OS) and progression-free survival. Results: Mean age was $68.7 \pm 9.2$ years and $59.4 \%$ patients were male. On multivariate analysis for OS, hypoalbuminemia and significant weight loss were prognostic at $p$-values of 0.0005 and 0.0052 , respectively. We noted a parabolic association between age and OS $(p=0.026,0.0025)$. Conclusion: In our study, some malnutrition parameters were associated with decreased OS. U-shape relationship between age and OS noted here warrants further evaluation.

First draft submitted: 10 March 2020; Accepted for publication: 20 March 2020; Published online: 24 April 2020

Keywords: immunotherapy $\bullet$ malnutrition $\bullet$ non-small-cell lung cancer

Lung cancer continues to be the most commonly diagnosed and deadliest form of cancer worldwide, accounting for approximately 1.7 million deaths in 2018 alone [1]. In the USA, there was an estimated 228,150 new cases and 142,670 deaths associated with lung cancer in 2019 [2]. Checkpoint inhibitor (CPI) therapy, referred to commonly as immunotherapy, has transformed the outcomes for advanced cancer patients over the last decade and has become an integral component of 'standard of care' treatment regimens for several types of cancers, including lung cancer [3]. Currently approved CPIs inhibit CTLA4, PD-1 or PD-L1. PD-1 and PD-L1 inhibitors have shown improved responses, progression-free survival (PFS) and overall survival (OS) in lung cancer patients compared with previous, 
'standard of care' chemotherapy treatments [4-12]. However, the benefit does not extend to all patients even when enriched for extensively studied predictive markers [13]. Some patients experience no benefit at all, and some suffer from a detrimental effect on their overall health, either due to serious immune-related adverse effects [14] or the phenomenon of hyperprogression [15], in addition to an incremental increase on financial burden. Therefore, it is imperative to carefully select patients who are likely to benefit for CPI immunotherapy. Significant research efforts toward identifying optimal biomarkers that predict outcomes to CPIs are currently underway $[16,17]$. Two such biomarkers that have been extensively studied and shown to have some predictive value in lung cancer, include tumor expression of PD-L1 and tumor mutational burden [16,17]. However, both of these biomarkers are tissuebased and are imperfect in dichotomizing patients who benefit and those who do not. Many other biomarkers that are currently being studied require tumor tissue specimens or involve cumbersome and complicated laboratorybased testing that can only be done at highly specialized centers and will likely be of limited use due to cost constraints [18]. Hence, it would be appropriate to identify readily available clinical and laboratory parameters that might help predict response to CPIs.

Hypothesis-generating preclinical observations suggested that baseline nutritional status may impact antitumor immunity $[19,20]$. There is also clinical data showing association between malnutrition and worse outcomes from CPI [21], and paradoxically, between obesity and improved benefit from immunotherapy [22]. Optimal assessment of nutritional status however is still being debated [23]. In this retrospective study, we looked at predictive and prognostic value of several factors hypothesized to be associated with nutritional status. Our study aimed to correlate malnutrition status prior to CPI initiation, BMI serum albumin and significant weight loss at presentation with OS and PFS.

\section{Materials \& methods \\ Study design}

This was an institutional review board approved retrospective chart review conducted at an academic medical center. Patients included in the study were adult advanced non-small-cell lung cancer (NSCLC) patients ( $\geq 18$ years of age) who received CPI, specifically monoclonal antibodies to PD-1 and PD-L1 as monotherapy in any line of treatment, between January 2014 and December 2017. Patients who had received CPI previously and those with no documented baseline albumin were excluded.

Electronic medical records were accessed to collect data on patient demographics, cancer details, details about immunotherapy treatment, details about prior chemotherapy regimens and smoking history. In addition, we captured data on Eastern Cooperative Group (ECOG) performance status (PS), BMI and serum albumin at the time of presentation. We also noted if there was a significant weight loss $(\geq 5 \%)$ reported within 6 months prior to presentation. Information on adverse events and their grades was also collected. In addition, dates of death, progression and last follow-up were recorded. Study data were collected and managed using Research Electronic Data Capture (REDCap) electronic data capture tools hosted at Feinstein Institutes for Medical Research. REDCap is a secure, web-based application designed to support data capture for research studies, providing an intuitive interface for validated data entry, audit trails for tracking data manipulation and export procedures, automated export procedures for seamless data downloads to common statistical packages and procedures for importing data from external sources [24].

\section{Statistical methods}

Malnutrition status was defined as BMI $<18.5$ and/or serum albumin $<3.5 \mathrm{mg} / \mathrm{dl}$ prior to CPI initiation. BMI was categorized and evaluated as a binary variable (obese vs nonobese) and as a three-level categorical variable: underweight/normal weight (BMI <25), overweight (BMI 25-30) and obese (BMI >30); due to few patients being classified as underweight (BMI $<18)$, these patients were combined with those of normal weight for analysis. Serum albumin level was evaluated as a binary variable using a cutoff of $<3.5 \mathrm{mg} / \mathrm{dl}$ (herein referred to as hypoalbuminemia) versus $\geq 3.5 \mathrm{mg} / \mathrm{dl}$. Significant weight loss at presentation was defined as $\geq 5 \%$ weight loss within 6 months prior to presentation.

Descriptive statistics (e.g., frequencies and proportions for categorical variables and means and standard deviations for continuous factors) were computed. OS and PFS were each analyzed by applying standard methods of survival analysis, specifically computation of Kaplan-Meier (K-M) product limit curves [25] and performance of Cox proportional hazards $(\mathrm{PH})$ regression [26]. In K-M analyses, groups were compared using the log-rank test. The median survival rates for each group were obtained from the K-M/Product-Limit Estimates and their corresponding 
95\% CIs were computed, as appropriate. Time-to-event was measured as the number of months from initial treatment (time from first dose of immunotherapy) to the event end point (progression or death). In cases, where the event was not observed during the study time period, the number of months until last follow-up was used and subject's survival status was classified as censored at that time point. For OS, the event end point was death and for PFS, the event was defined as either progression or death.

For all analyses, where appropriate, demographic or clinical factors that appeared to be significantly associated with each outcome in the univariate analysis at alpha level 0.1 , were considered for inclusion in a multivariable Cox $\mathrm{PH}$ model. Appropriate functional form of continuous factors and $\mathrm{PH}$ assumption for models were assessed and corrected as applicable. Multivariable model selection was performed using the likelihood ratio test, or backward elimination using 5\% level of significance, as appropriate, and the model with the lowest Akaike Information Criteria (AIC) [27] was chosen when comparing non-nested models. For all analyses, a result yielding $\mathrm{p}<0.05$ was considered statistically significant unless stated otherwise. All analyses were conducted using SAS version 9.4 (SAS Institute Inc., NC, USA). Immune-related adverse events of all grades and serious adverse events for the entire cohort was tabulated.

\section{Results}

A total of 115 patients were identified and 9 patients were excluded for missing baseline albumin values, leaving 106 patients included for the final analysis. Most of the patients $(n=65)$ had de novo Stage IV disease based on American Joint Cancer Committee (AJCC), edition 7, while 30 patients had recurrent or progressive disease after being previously treated for Stage III $(n=24)$, Stage II $(n=5)$ and Stage $1(n=2)$. For 10 patients, initial staging was not recorded. The mean age was $68.7 \pm 9.2$ years and 63 patients $(59.4 \%)$ were male. $68 \%$ of the patients were Caucasian White, while 18,6 and 14\% were African American, Asian and unknown race, respectively. Approximately $85 \%$ of patients had anytime history of smoking, and $21.7 \%$ were active smokers at the time of treatment initiation. Based on previously established cutoffs for BMI [28], 8.5, 28.3, 38.7 and 24.5\% were classified as underweight, normal, overweight and obese, respectively. $17 \%$ of patients had a low albumin $(<3.5 \mathrm{~g} / \mathrm{dl})$ at baseline and $37.7 \%$ reported weight loss of $5 \%$ or more in the 6 months prior to starting immunotherapy. 23 $(21.7 \%)$ of the patients were malnourished based on the composite nutritional assessment that included BMI and baseline serum albumin level. About 17, 49, 25 and 8.7\% of patients had a baseline ECOG PS of 0, 1, 2 and 3, respectively. 59 (56\%) patients received nivolumab, 25 (24\%) received pembrolizumab, 21 (20\%) received atezolizumab and $1(0.9 \%)$ received avelumab. About 16, 64.1 and 14.2\%, of patients received immunotherapy in the first-, second- and third-line setting, while 5.7\% of patients had received more than three lines of prior treatment. The number of immunotherapy cycles received ranged from 1 to 36 with the median number of cycles being 6 (interquartile range: 4-12). 47 (44.3\%) patients had also received thoracic radiation previously. Table 1 provides complete descriptive statistics on the subjects included in this study.

OS

Of 106 subjects, death was observed in 43 subjects. The median OS was 16.3 months (95\% CI: 10.4-22.2). The estimated survival probability at 12 and 24 months after initiating immunotherapy were 55.2\% (95\% CI: 42.8-66.0) and 22.0\% (95\% CI: 7.4-41.4), respectively. Median OS comparisons between subsets of patients based on various nutritional assessment parameters are depicted in Table 2.

None of the following factors: race, ethnicity, BMI, smoking status or gender, were found to be significantly associated with OS on univariate analysis. Low serum albumin level status, malnutrition status based on composite nutritional assessment, report of at least 5\% weight loss within 6 months of presentation, and baseline ECOG PS were each significantly associated with OS on univariate screen $(p<0.0001, p=0.0001, p=0.0003$, $\mathrm{p}=0.0162$, respectively). Age was also marginally associated with OS on univariate analysis $(\mathrm{p}=0.06)$. Due to the multicollinearity and correlation concerns between malnutrition status and albumin, each of these factors were entered into separate multivariable Cox PH models, along with, ECOG score, age and initial $\geq 5 \%$ weight loss status within 6 months of initiation of CPI. The final multiple Cox PH regression model included low serum albumin level status, initial 5\% weight loss and age as a quadratic term (see Figures 1 \& 2, Table 3). On multivariate analysis, an interesting U-shaped relationship was noted between age and OS (Figure 2). Risk of death was highest at lower ages, insignificant at 'middle' ages (i.e., ages 60-75), and at the lowest for elderly patients (i.e., $>75$ years of age). Our observations seem to suggest that patients above the estimated age of 75 years have an incrementally 


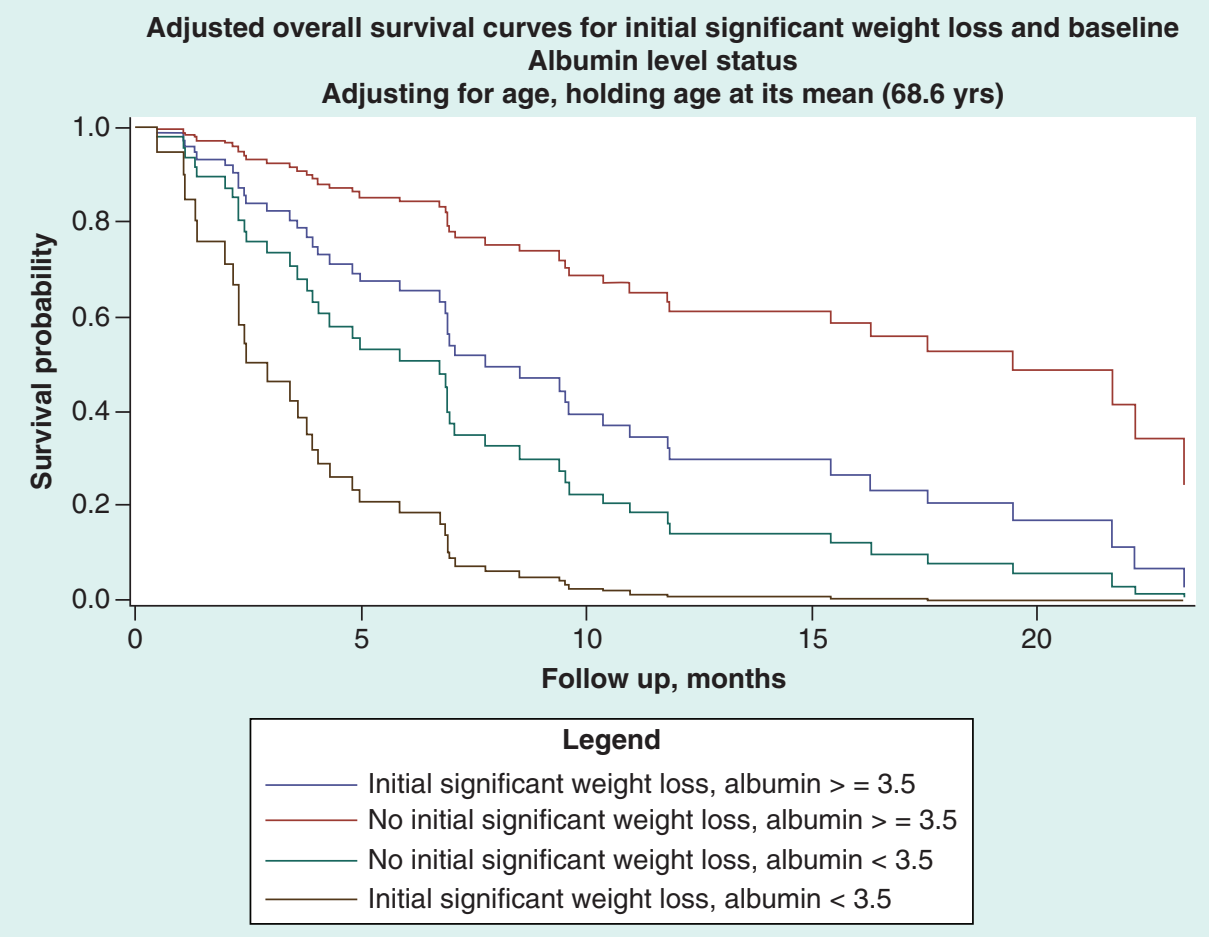

Figure 1. Adjusted overall survival curves for initial significant weight loss and baseline albumin level status.

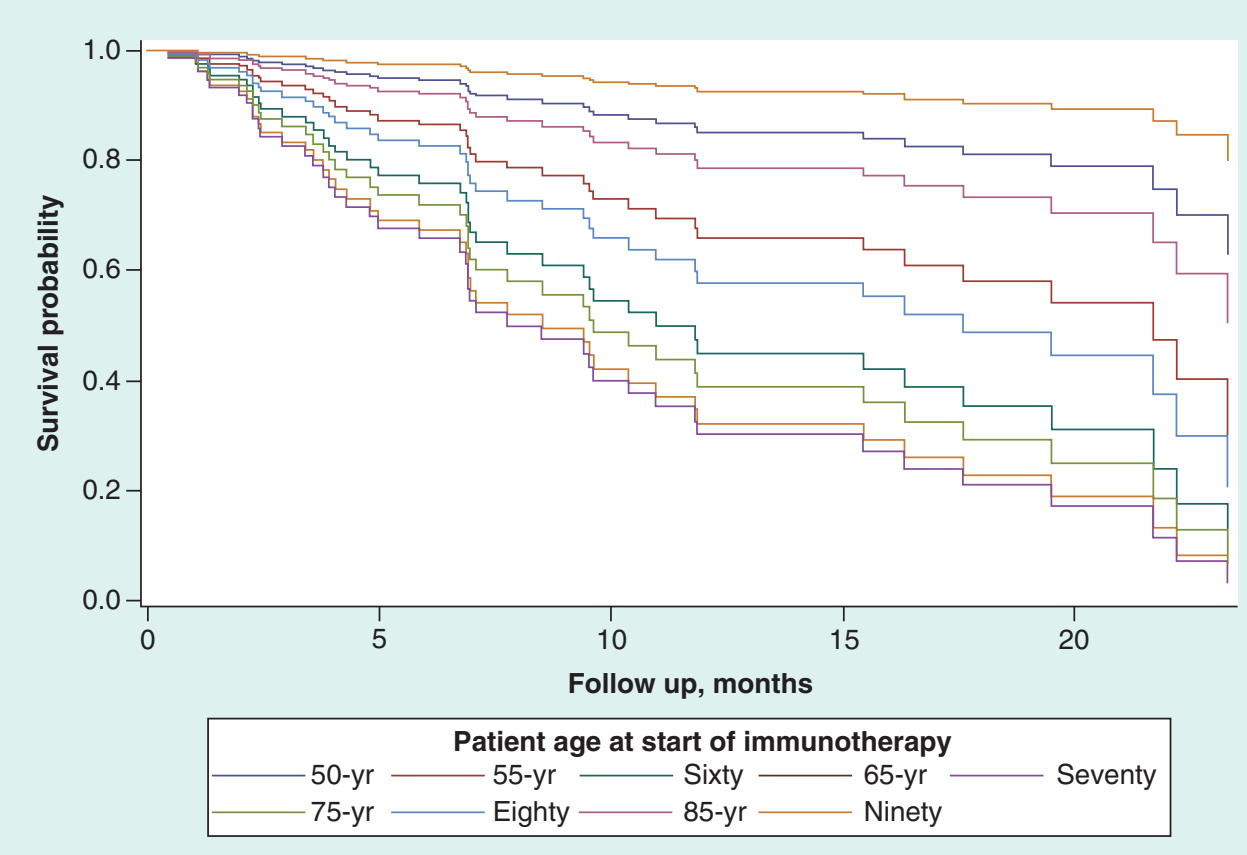

Figure 2. Predicted overall survival curves for various patient age profiles, holding serum albumin $\geq 3.5 \mathrm{~g} / \mathrm{dl}$ and reported $\geq 5 \%$ weight loss within 6 months prior to immunotherapy initiation. 


\begin{tabular}{|c|c|}
\hline Baseline characteristic & Total $(n=106)$ \\
\hline Age (years) & $68.6 \pm 9.2$ \\
\hline Male & $63(59.4 \%)$ \\
\hline Female & $43(41.6 \%)$ \\
\hline $\begin{array}{l}\text { Race: } \\
\text { - White } \\
\text { - Black } \\
\text { - Asian } \\
\text { - Unknown }\end{array}$ & $\begin{array}{l}68(64.2 \%) \\
18(17.0 \%) \\
6(5.7 \%) \\
14(13.2 \%)\end{array}$ \\
\hline BMI & $25.7 \pm 5.4$ \\
\hline $\begin{array}{l}\text { Smoking status: } \\
\text { - Yes } \\
\text { - Former }\end{array}$ & $\begin{array}{l}23(21.7 \%) \\
67(63.2 \%)\end{array}$ \\
\hline Baseline albumin & $3.9 \pm 0.4$ \\
\hline Baseline albumin $(<3.5 \mathrm{~g} / \mathrm{dl})$ & $18(17 \%)$ \\
\hline Malnourished based CNA & $23(21.7 \%)$ \\
\hline Initial $\geq 5 \%$ weight loss upon starting immunotherapy & $40(37.7 \%)$ \\
\hline $\begin{array}{l}\text { ECOG PS: } \\
-0 \\
-1 \\
-2 \\
-3\end{array}$ & $\begin{array}{l}18(17.3 \%) \\
51(49.0 \%) \\
26(25.0 \%) \\
9(8.7 \%)\end{array}$ \\
\hline $\begin{array}{l}\text { Line of therapy } \\
\text { - First } \\
\text { - Second } \\
\text { - Third or more }\end{array}$ & $\begin{array}{l}17(16.0 \%) \\
68(64.2 \%) \\
21(19.8 \%)\end{array}$ \\
\hline $\begin{array}{l}\text { Morphological classification } \\
\text { - Adenocarcinoma } \\
\text { - Squamous } \\
\text { - Small cell }\end{array}$ & $\begin{array}{l}68(67.3 \%) \\
25(24.8 \%) \\
8(7.9 \%)\end{array}$ \\
\hline Radiation during immunotherapy & $10(9.4 \%)$ \\
\hline Number of cycles, median (IQR) & $4(6-12)$ \\
\hline
\end{tabular}

\begin{tabular}{|c|c|c|c|c|c|}
\hline Variables & $\mathbf{N}$ & Median OS, months $(95 \% \mathrm{Cl})$ & p-value & Median PFS, months (95\% Cl) & p-value \\
\hline \multicolumn{6}{|l|}{ Composite nutritional status } \\
\hline Malnutrition status & 23 & $7.1(4.8-10.4)$ & 0.0001 & $6.3(4.1-9.9)$ & 0.25 \\
\hline Normal nutritional status & 83 & 21.7 (11.9 to not estimable) & & $8.5(5.6-16.5)$ & \\
\hline \multicolumn{6}{|l|}{ BMI } \\
\hline Underweight/Normal BMI & 50 & $7.3(5.6-15.9)$ & 0.4 & $19.5(9.6-23.3)$ & 0.98 \\
\hline Overweight & 30 & 8.5 (3.5 to not estimable) & & Not estimable & \\
\hline Obese & 26 & $11.9(6.9-17.6)$ & & $9.4(4.1-17.3)$ & \\
\hline \multicolumn{6}{|l|}{ Weight loss } \\
\hline Weight loss $>5 \%$ & 40 & $6.1(4.0-17.6)$ & 0.0003 & $7.4(4.6-15.9)$ & 0.75 \\
\hline No weight loss $>5 \%$ & 66 & 21.7 (11.9 to not estimable) & & $8.5(5.6-16.5)$ & \\
\hline \multicolumn{6}{|l|}{ Albumin } \\
\hline$<3.5 \mathrm{~g} / \mathrm{dl}$ & 18 & $6.9(2-10.4)$ & 0.00001 & $5.2(3.9-9.4)$ & 0.0792 \\
\hline$\geq 3.5 \mathrm{~g} / \mathrm{dl}$ & 88 & $19.5(11.9-23.3)$ & & $8.5(5.6-15.9)$ & \\
\hline
\end{tabular}

improved survival with every 5-year increase in age at the time of initiation of CPI, whereas the risk of death increases with every 5 -year increase in age from 45 to 60 years. 
Table 3. Final cox proportional hazard model for mortality.

\begin{tabular}{|c|c|c|c|c|c|c|}
\hline Parameter & $\begin{array}{l}\text { Parameter } \\
\text { Estimate }\end{array}$ & Standard Error & p-value & Hazard Ratio & & $6 \mathrm{Cl}$ \\
\hline $\begin{array}{l}\text { Was there initial }(\geq 5 \%) \text { weight loss upon starting } \\
\text { immunotherapy? } \\
\text { Yes vs No }\end{array}$ & 0.91 & 0.32 & 0.0052 & 2.48 & 1.31 & 4.68 \\
\hline Albumin $<3.5 \mathrm{~g} / \mathrm{dl}$ & 1.37 & 0.39 & 0.0005 & 3.95 & 1.83 & 8.54 \\
\hline Age when started immunotherapy & 0.81 & 0.36 & 0.026 & - & - & - \\
\hline $\mathrm{Age}^{\dagger}$ & -0.01 & 0.00 & 0.0249 & - & - & - \\
\hline
\end{tabular}

PFS

Among 106 subjects, progression events were observed in 50 subjects, with five deaths occurring before progression could be observed, with the remaining 51 subjects' observations censored. The median time of PFS was 7.4 months (95\% CI: 5.6-12.1). The estimated PFS probability at 12 months after initiating immunotherapy was 39.7\% $(95 \%$ CI: 27.9-51.3). The estimated PFS probability at 18 months after initiating immunotherapy was $17.3 \%$ (95\% CI: 11.5-39.3).

None of the following factors were found to have significant association with PFS: race, ethnicity, BMI, obesity, smoking status, gender, ECOG PS, malnutrition status, presence of at least $5 \%$ initial weight loss at presentation or age $(\mathrm{p}=0.7, \mathrm{p}=0.27, \mathrm{p}=0.98, \mathrm{p}=0.96, \mathrm{p}=0.88, \mathrm{p}=0.87, \mathrm{p}=0.77, \mathrm{p}=0.25, \mathrm{p}=0.74$ and $\mathrm{p}=0.76$, respectively). Serum albumin less than 3.5 at baseline was associated with PFS on univariate screen at $\mathrm{p}<0.1$ level $(p=0.079)$. Given that no factor was significantly associated with PFS other than low albumin status from univariate screen, a multivariable Cox PH model was not built.

\section{Adverse events}

Overall, 96 patients (90\%) experienced an adverse event, with only three patients $(2.8 \%)$ experiencing a severe event. Fatigue/weakness (54.7\%), dyspnea (21.7\%), rash/pruritus (16\%), diarrhea/colitis (15.1\%) and nausea/vomiting (13.2\%) were the most common adverse events. A total of 20 patients $(21.7 \%)$ discontinued CPI due to adverse events and 5 patients (4.7\%) required hospital admission. The incidence of adverse events of all grades and severe adverse events are depicted in Table 4.

\section{Discussion}

The relationship between nutritional status and homeostasis of the immune system and anticancer immunity is a complex one and recent research into this field is shedding some light into this very intriguing association [19,20,29-32] . Advanced cancer is associated with a cancer-induced wasting syndrome, also called cancer cachexia, which results in caloric deficiency [33]. Caloric deficiency, in turn is linked to immunosuppression, increased susceptibility to infection and has been shown to be associated with cancer progression but somewhat protective against autoimmune diseases [19,31]. Obesity on the other hand is associated with an overactive immune system, auto-immunity and enhanced anticancer immunity [32,34,35]. Nutritional status has influence on T-cell metabolism and function through mediation by several inter-related cytokines such as IL- 6 and TNF- $\alpha$ as well as stress hormones [19]. In mice models, both TNF- $\alpha$ and IL- 6 were noted to be markedly elevated in precachexic and cachexic states of cancer patients $[19,36$. One of the many possible mechanisms by which IL-6 interplays with tumor immunity is its involvement in decreasing hepatic ketogenic potential through suppression of PPARalpha [19]. Hypoketonemia which can be considered a systemic metabolic stress response resulting from precachectic and cachectic states in turn triggers a marked elevation in glucocorticoid levels, which has been in turn been shown to be associated with poor response to immunotherapy and overall poor outcomes [19]. Contrariwise, obesity has shown to be associated with improved responses to CPI in multiple recent studies [20,22,34,37,38]. The mechanistic explanation for this association is currently being elucidated but there are some hypotheses. Macrophages are the most abundant immune cells in adipose tissues and during periods of weight gain, there is an influx of inflammatory macrophages into the adipose tissue and this leads release of cytokines such as TNF- $\alpha$ [20,39]. Thus, obesity results in heightened inflammatory response that interferes with immune modulation, inhibits antitumor immunity and results in immune exhaustion. CPI therapy seems to reverse this process and enhance antitumor immunity. 


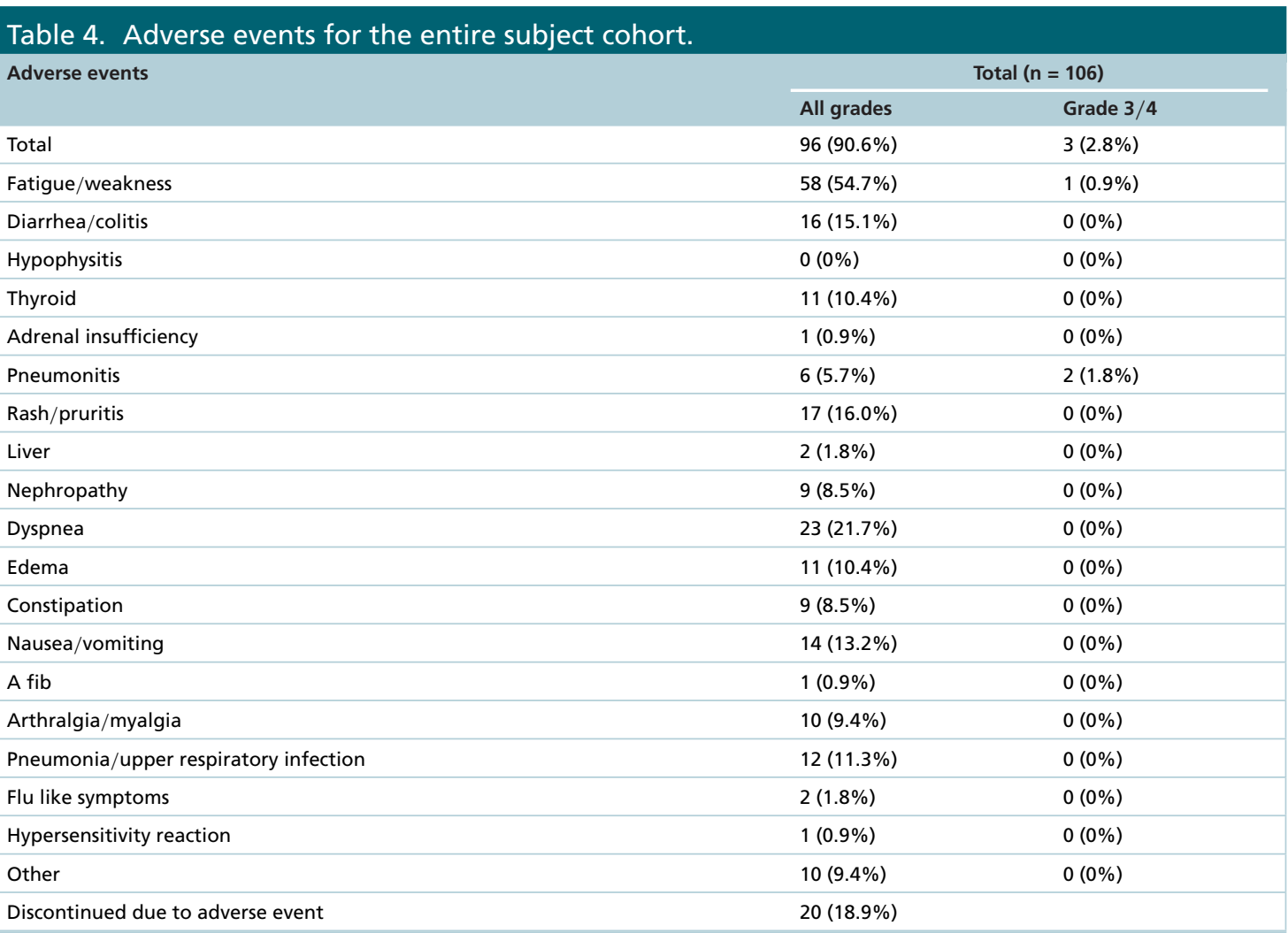

While there is a now general agreement that nutritional status is an important factor in predicting responses to CPI, there has been considerable variability when it comes to methods used for assessment of nutritional status [23]. Common tools include subjective quantification of weight loss by patients [40]; objective anthropometric measurements such as BMI [41,42]; skinfold thickness [43] and laboratory assessments such as serum CRP [44], and pre-treatment serum albumin [44,45] and dietary assessments such as 24-h food intake recall [46]; and body composition assessment such as lean muscle mass area calculated from axial-view computerized tomographic images of the abdomen at the level of L3 spine [47]. Combining various parameters is sometimes utilized for nutritional assessment and one of such composite measure is integration of BMI and serum albumin which has been validated in patients on hemodialysis and cancer [48]. In our study, we utilized nutritional parameters that were readily available from a retrospective chart review. We chose weight loss of 5\% or more in the 6 months preceding CPI therapy, BMI, obesity, composite assessment of malnutrition using BMI and serum albumin as our primary measures of nutritional status.

Velocity of weight loss has been historically used as a measure for malnutrition and the velocity of weight loss has been shown to correspond with other cancer related symptoms such as anorexia and fatigue as well as disease outcomes [40]. Weight loss by itself as a measure of nutrition is flawed since this is in many cases, this is subjective and dependent on patients' recall. It does not discriminate between intentional and unintentional weight loss. In our study, there was a significant association between having a significant unintentional weight loss $(5 \%$ or more in 6 months) and decreased OS probability.

BMI has been shown to be predictive of outcomes in several disease conditions such as Type 2 diabetes [49]. It has associated with increased risk for developing certain cancers such as that of colon and endometrium [34]. Despite this, using BMI as a sole measure of nutritional status has several pitfalls: BMI cannot distinguish fat and lean masses, and it may be particularly in cancer patients may have a relative increase in body fat and fluids, and a severe decline in lean tissue [50]. In our study, BMI was not associated with OS or PFS. We did not observe improved outcomes associated with overweight and obese status in our patient population. While this might have simply been a reflection of the small size of our study population, it may also support previous reports of BMI as an inaccurate measure of nutritional status. 
Albumin is a protein synthesized in the liver and has a half-life of 14-20 days, making serum albumin an easy, stable measure for nutritional status [45]. However, albumin levels are easily affected by its levels are affected by inflammation, liver dysfunction and many other conditions in addition to nutritional status. In our study, we found that the serum albumin less than 3.5 was the only factor that was possibly associated with worse OS and PFS probabilities.

Theoretically, combining with BMI and albumin might be a more efficient tool to assess nutrition because they are likely to complement each other. For example, in patients with falsely elevated BMI because of increased fluids either from third-spacing or effusions, low serum albumin may point toward malnutrition. This composite measure has been validated in prior studies [43]. In our study, we used albumin cutoff of less than 3.5 and/or BMI $<18.5$ as a marker of malnutrition status. This composite score was significantly associated with OS in univariate screen. However, this measure was not incorporated into the final Cox-proportionate multivariable model selection, due to multicollinearity and correlation concerns if both malnutrition status and albumin were to be included. The model with low serum albumin status was chosen as having the best model fit.

An interesting and unexpected quadratic relationship between age and survival was observed in our study. This suggests that for our cohort, younger age is associated with greatest hazard of death at earlier time points, which declines with increasing age. In the middle age range of the cohort, the effect of age on survival is not meaningful. However, as age at start of therapy continues at about 75 years or older, the effect of age on survival seems to improve (risk of death decreases). To better understand the clinical background and biologic rationale of the relationship between age and clinical outcomes to CPI, we reviewed relevant literature on the use of immunotherapy in elderly patients with cancer.

Counterintuitive to expectations that CPI would be ineffective in elderly patients with cancer due to immunesenescence, which is an age-related decline in the immune function [51], recent studies have shown that age is not an adverse predictive factor for responses to CPI and may in fact be a favorable predictive factor for patients on CPI. In a retrospective cohort study of 500 patients with metastatic melanoma treated with PD1 inhibitors as monotherapy, 144 patients who were $\geq 65$ years age were selected for further analysis. They were then stratified into two age groups of 65-79 years and 80-100 years. Complete response rates were significantly higher for the older cohort of patient ( 48 vs $20 \% ; \mathrm{p}=0.001$ ) and overall response also trended higher as compared with the younger patients [52]. Safety analysis showed similar profiles of immune-related adverse events, and similar rates of grade 3 and 4 toxicity.

Another retrospective analysis of 246 patients focused solely on a population of patients with NSCLC who received PD-L1 inhibitors [53]. Patients in this study were divided into age groups of $<60,60-69,70-79$ and $\geq 80$ years. The findings of this study differed a little from the aforementioned study in that patients $<60$ years had a less favorable survival outcome. With increasing age, outcomes improved and then worsened again after age 80 . One possible explanation for the somewhat contrasting finding between these studies is the inherent differences between the patient populations in the two studies. It is possible, that elderly patients with lung cancer age have higher prevalence of co-morbid conditions [54], such as heart and lung diseases which become a source of competing mortality. Also, the age range in the former study contained very elderly individuals, well past 80 years of age. Additional methodological issues with these studies including selection bias and unmeasured confounders, might have contributed to the minor differences between the study results. A meta-analysis of nine randomized controlled clinical trials was performed on a total of 5265 patients who received CPIs for various indications, including lung cancer, melanoma and renal cancer [55]. Patients were divided into younger and older groups with an age cutoff for subgroup analysis of 65-70 years, CPI improved OS in both the younger (HR: 0.75; 95\% CI: 0.68-0.82) and older (HR: 0.73; 95\% CI: 0.62-0.87) groups. Since this meta-analysis included mostly melanoma randomized controlled trials, its generalizability to lung cancer is limited. Nevertheless, all these studies support activity and safety of CPI in the elderly patient population and our study echoed the same finding by demonstrating an interesting U-shape relationship between age and CPI outcomes. A plausible explanation for this rather paradoxical observation was explored in preclinical studies. In a melanoma mouse model [56], response to PD1 inhibition was significantly worse for younger ( 2 months) mice compared with older (10 months) ones. The study suggested that one explanation for this observation may be because of increase in immunosuppressive FOXP3 ${ }^{+}$Tregs within the tumor microenvironment and decrease in $\mathrm{CD}^{+} \mathrm{T}$ effector cells, which serve as the target of PDL1 inhibition in the younger mice. 


\section{Limitations}

There are several limitations to our study. First, this study was retrospective in nature. Furthermore, due to the limited sample size, it is possible that there was insufficient power to detect small, yet clinically meaningful differences in PFS probabilities for other risk factors. Third, as previously mentioned some of the limitations of using BMI as a sole measure of nutritional status, it was felt that albumin would be a good complement to assess nutritional status.

\section{Conclusion}

Our study indicates that pretreatment nutritional status is associated with OS and possibly with PFS as well for patients treated with CPI therapy. Optimal measure for nutritional status is unclear but based on our single institution retrospective review, serum albumin and significant weight loss prior to starting CPI were significantly associated with OS in multivariate analysis. We noted an interesting parabolic age-response correlation for patients with NSCLC treated with CPI, and this warrants further evaluation with a larger sample size and preferably in a prospective fashion.

\section{Future perspective}

Immunotherapy has changed the way we treat cancer and for some, long, durable responses have been shown. However, this benefit does not extend to all patients, with some patients experiencing no benefit at all and some suffering from a detrimental effect on their overall health either due to serious immune-related adverse effects. It is essential to carefully select patients who are likely to benefit for CPI immunotherapy. Significant research efforts toward identifying optimal biomarkers that predict outcomes to CPIs are currently underway. Nutritional status may play a role in antitumor immunity, but is still being debated. Further research will provide additional, much needed insight on this topic.

\section{Author contributions}

All authors contributed to the study design and putting together the manuscript.

\section{Acknowledgments}

The authors would like to thank R Sin for her assistance with data collection.

Financial \& competing interests disclosure

$\mathrm{N}$ Seetharamu has served on the advisory boards for Genentech and AstraZeneca in the last year. The authors have no other relevant affiliations or financial involvement with any organization or entity with a financial interest in or financial conflict with the subject matter or materials discussed in the manuscript apart from those disclosed.

No writing assistance was utilized in the production of this manuscript.

\section{Open access}

This work is licensed under the Attribution-NonCommercial-NoDerivatives 4.0 Unported License. To view a copy of this license, visit http://creativecommons.org/licenses/by-nc-nd/4.0/

\section{References}

Papers of special note have been highlighted as: • of interest; $\bullet \bullet$ of considerable interest

1. Bray F, Ferlay J, Soerjomataram I, Siegel RL, Torre LA, Jemal A. Global cancer statistics 2018: GLOBOCAN estimates of incidence and mortality worldwide for 36 cancers in 185 countries. CA Cancer J. Clin. 68(6), 394-424 (2018).

2. Siegel RL, Miller KD, Jemal A. Cancer statistics, 2019. CA Cancer J. Clin. 69(1), 7-34 (2019).

3. Gong J, Chehrazi-Raffle A, Reddi S, Salgia R. Development of PD-1 and PD-L1 inhibitors as a form of cancer immunotherapy: a comprehensive review of registration trials and future considerations. J. Immunother. Cancer 6(1), 8 (2018).

4. Brahmer J, Reckamp KL, Baas $\mathrm{P}$ et al. Nivolumab versus docetaxel in advanced squamous-cell non-small-cell lung cancer. N. Engl. J. Med. 373(2), 123-135 (2015).

5. Ready N, Hellmann MD, Awad MM et al. First-line nivolumab plus ipilimumab in advanced non-small-cell lung cancer (CheckMate 568): outcomes by programmed death ligand 1 and tumor mutational burden as biomarkers. J. Clin. Oncol. 37(12), 992-1000 (2019).

6. Borghaei H, Paz-Ares L, Horn L et al. Nivolumab versus docetaxel in advanced nonsquamous non-small-cell lung cancer. N. Engl. J. Med. 373(17), 1627-1639 (2015).

7. Paz-Ares L, Luft A, Vicente D et al. Pembrolizumab plus chemotherapy for squamous non-small-cell lung cancer. N. Engl. J. Med. 379(21), 2040-2051 (2018). 
8. Herbst RS, Baas P, Kim DW et al. Pembrolizumab versus docetaxel for previously treated, PD-L1-positive, advanced non-small-cell lung cancer (KEYNOTE-010): a randomised controlled trial. Lancet 387(10027), 1540-1550 (2016).

9. Garon EB, Rizvi NA, Hui R et al. Pembrolizumab for the treatment of non-small-cell lung cancer. N. Engl. J. Med. 372(21), 2018-2028 (2015).

10. Socinski M, Creelan B, Horn L et al. CheckMate 026: a Phase III trial of nivolumab vs investigator's choice (IC) of platinum-based doublet chemotherapy (PT-DC) as first-line therapy for stage iv/recurrent programmed death ligand 1 (PD-L1)-positive NSCLC. Ann. Oncol. 27(Suppl. 6), vi552-vi587 (2016).

11. Mok TSK, Wu Y-L, Kudaba I et al. 102OFinal analysis of the Phase III KEYNOTE-042 study: pembrolizumab (Pembro) versus platinum-based chemotherapy (Chemo) as first-line therapy for patients (Pts) with PD-L1-positive locally advanced/metastatic NSCLC. Ann. Oncol. 30(2), i38 (2019).

12. Langer CJ, Gadgeel SM, Borghaei H et al. Carboplatin and pemetrexed with or without pembrolizumab for advanced, non-squamous non-small-cell lung cancer: a randomised, phase 2 cohort of the open-label KEYNOTE-021 study. Lancet Oncol. 17(11), 1497-1508 (2016).

13. Shukuya T, Carbone DP. Predictive markers for the efficacy of anti-PD-1/PD-L1 antibodies in lung cancer. J. Thorac. Oncol. 11(7), 976-988 (2016).

14. Postow MA, Sidlow R, Hellmann MD. Immune-related adverse events associated with immune checkpoint blockade. N. Engl. J. Med. 378(2), 158-168 (2018).

15. Fuentes-Antrás J, Provencio M, Díaz-Rubio E. Hyperprogression as a distinct outcome after immunotherapy. Cancer Treat. Rev. 70, 16-21 (2018).

16. Samstein RM, Lee CH, Shoushtari AN et al. Tumor mutational load predicts survival after immunotherapy across multiple cancer types. Nat. Genet. 51(2), 202-206 (2019).

17. Jacquelot N, Roberti MP, Enot DP et al. Predictors of responses to immune checkpoint blockade in advanced melanoma. Nat. Commun. 8(1), 592 (2017).

18. Otoshi T, Nagano T, Tachihara M, Nishimura Y. Possible biomarkers for cancer immunotherapy. Cancers (Basel) 11(7), 935 (2019).

19. Flint TR, Janowitz T, Connell CM et al. Tumor-induced IL-6 reprograms host metabolism to suppress anti-tumor immunity. Cell Metab. 24(5), 672-684 (2016).

20. Wang Z, Aguilar EG, Luna JI et al. Paradoxical effects of obesity on T cell function during tumor progression and PD-1 checkpoint blockade. Nat. Med. 25(1), 141-151 (2019).

- Indicates a paradoxical impact of obesity on cancer and that obesity is associated with increased efficacy of program death-1/program death ligand-1 blockade in tumor-bearing mice and clinical cancer patients.

21. Coss CC, Clinton SK, Phelps MA. Cachectic cancer patients: immune to checkpoint inhibitor therapy? Clin. Cancer Res. 24(23),5787 5789 (2018).

- Cachectic cancer patients exhibit poorer response to immunotherapy.

22. Murphy WJ, Longo DL. The surprisingly positive association between obesity and cancer immunotherapy efficacy. JAMA 321(13), 1247-1248 (2019).

23. Andreoli A, De Lorenzo A, Cadeddu F, Iacopino L, Grande M. New trends in nutritional status assessment of cancer patients. Eur. Rev. Med. Pharmacol. Sci. 15(5), 469-480 (2011).

24. Harris PA, Taylor R, Thielke R, Payne J, Gonzalez N, Conde JG. Research electronic data capture (REDCap)-A metadata-driven methodology and workflow process for providing translational research informatics support. J. Biomed. Inform. 42(2), 377-381 (2009).

25. Efron B. Logistic regression, survival analysis, and the kaplan-meier curve. J. Am. Stat. Assoc. 83(402), 414-425 (1988).

26. Cai J, Zeng D. Cox proportional hazard model. In: Wiley Encyclopedia of Clinical Trials. D’agostino R, Massaro J, Sullivan L (Eds). John Wiley \& Sons, Inc, NJ, USA (2008).

27. Cavanaugh JE. Unifying the derivations for the Akaike and corrected Akaike information criteria. Stat. Probab. Lett. 33(2), 201-208 (1997).

28. Flegal KM, Kit BK, Graubard BI. Body mass index categories in observational studies of weight and risk of death. Am. J. Epidemiol. 180(3), 288-296 (2014).

29. Soldati L, Di Renzo L, Jirillo E, Ascierto PA, Marincola FM, De Lorenzo A. The influence of diet on anti-cancer immune responsiveness. J. Transl. Med. 16(1), 75 (2018).

30. Zitvogel L, Pietrocola F, Kroemer G. Nutrition, inflammation and cancer. Nat. Immunol. 18(8), 843-850 (2017).

31. Pae M, Meydani SN, Wu D. The role of nutrition in enhancing immunity in aging. Aging Dis. 3(1), 91-129 (2012).

32. Valdés-Ramos R, Benítez-Arciniega AD. Nutrition and immunity in cancer. Br. J. Nutr. 98(S1), S127-S132 (2007).

33. Thoresen L, Frykholm G, Lydersen $S$ et al. Nutritional status, cachexia and survival in patients with advanced colorectal carcinoma. Different assessment criteria for nutritional status provide unequal results. Clin. Nutr. 32(1), 65-72 (2013). 
34. Wolin KY, Carson K, Colditz GA. Obesity and cancer. Oncologist 15(6), 556-565 (2010).

35. Versini M, Jeandel PY, Rosenthal E, Shoenfeld Y. Obesity in autoimmune diseases: not a passive bystander. Autoimmun. Rev. 13(9), 981-1000 (2014)

36. Landskron G, De La Fuente M, Thuwajit P, Thuwajit C, Hermoso MA. Chronic inflammation and cytokines in the tumor microenvironment. J. Immunol. Res. doi:10.1155/2014/149185 (2014) (Epub ahead of print).

37. Canter RJ, Le CT, Beerthuijzen JMT, Murphy WJ. Obesity as an immune-modifying factor in cancer immunotherapy. J. Leukoc. Biol. 104(3), 487-497 (2018).

38. Lysaght J. The 'obesity paradox' in action with cancer immunotherapy. Nat. Rev. Endocrinol. 15, 132-133 (2019).

39. Mirsoian A, Bouchlaka MN, Sckisel GD et al. Adiposity induces lethal cytokine storm after systemic administration of stimulatory immunotherapy regimens in aged mice. J. Exp. Med. 211(12), 2373-2383 (2014).

40. Gioulbasanis I, Baracos VE, Giannousi Z et al. Baseline nutritional evaluation in metastatic lung cancer patients: mini nutritional assessment versus weight loss history. Ann. Oncol. 22(4), 835-841 (2011).

41. Cortellini A, Bersanelli M, Buti S et al. A multicenter study of body mass index in cancer patients treated with anti-PD-1/PD-L1 immune checkpoint inhibitors: when overweight becomes favorable. J. Immunother. Cancer 7(1), 57 (2019).

42. Ibrahimi S, Mukherjee S, Roman D, King C, Machiorlatti M, Aljumaily R. Effect of body mass index and albumin level on outcomes of patients receiving anti PD-1/PD-L1 therapy. J. Clin. Oncol. 36(Suppl. 5), 213 (2018).

43. Kalantar-Zadeh K, Kleiner M, Dunne E, Lee GH, Luft FC. A modified quantitative subjective global assessment of nutrition for dialysis patients. Nephrol. Dial. Transplant. 14(7), 1732-1738 (1999).

44. Banh L. Serum proteins as markers of nutrition: what are we treating? Pract. Gastroenterol. 30(10), 46-64 (2006).

45. Don BR, Kaysen G. Serum albumin: relationship to inflammation and nutrition. Semin. Dial. 17(6), $432-437$ (2004).

46. Castell GS, Serra-Majem L, Ribas-Barba L. What and how much do we eat? 24-hour dietary recall method. Nutr. Hosp. 31(Suppl. 3), 46-48 (2015).

47. Vandewoude MFJ, Alish CJ, Sauer AC, Hegazi RA. Malnutrition-sarcopenia syndrome: is this the future of nutrition screening and assessment for older adults? J. Aging Res. doi:10.1155/2012/651570 2012) (Epub ahead of print).

48. Leinig CE, Moraes T, Ribeiro S et al. Predictive value of malnutrition markers for mortality in peritoneal dialysis patients. J. Ren. Nutr. 21(2), 176-183 (2011).

49. Narayan KMV, Boyle JP, Thompson TJ, Gregg EW, Williamson DF. Effect of BMI on lifetime risk for diabetes in the U.S. Diabetes Care 30(6), 1562-1566 (2007).

50. Baracos VE, Reiman T, Mourtzakis M, Gioulbasanis I, Antoun S. Body composition in patients with non-small cell lung cancer: a contemporary view of cancer cachexia with the use of computed tomography image analysis. Am. J. Clin. Nutr. 91(4), S1133-S1137 (2010).

51. Kanesvaran R, Cordoba R, Maggiore R. Immunotherapy in older adults with advanced cancers: implications for clinical decision-making and future research. Am. Soc. Clin. Oncol. Educ. B 38, 400-414 (2018).

- Describes the role of immunotherapy in older adults with lung cancer, bladder cancer and lymphomas.

52. Ben-Betzalel G, Steinberg-Silman Y, Stoff R et al. Immunotherapy comes of age in octagenarian and nonagenarian metastatic melanoma patients. Eur. J. Cancer 108, 61-68 (2019).

- Increasing age within the elderly patients group may predict a better response to therapy and comparable survival in patients of very old age in the metastatic melanoma setting.

53. Lichtenstein MRL, Nipp RD, Muzikansky A et al. Impact of age on outcomes with immunotherapy in patients with non-small cell lung cancer. J. Thorac. Oncol. 14(3), 547-552 (2019).

-• In non-small-cell lung cancer patients treated with immunotherapy, outcomes improved with increasing age and then worsened again after age 80 .

54. Dutkowska AE, Antczak A. Comorbidities in lung cancer. Adv. Respir. Med. 84(3), 186-192 (2016).

55. Nishijima TF, Muss HB, Shachar SS, Moschos SJ. Comparison of efficacy of immune checkpoint inhibitors (ICIs) between younger and older patients: a systematic review and meta-analysis. Cancer Treat. Rev. 45, 30-37 (2016).

56. Kugel CH, Douglass SM, Webster MR et al. Age correlates with response to anti-PD1, reflecting age-related differences in intratumoral effector and regulatory T-cell populations. Clin. Cancer Res. 24(21), 5347-5356 (2018). 
